\title{
Blood component treatment: a retrospective audit in five major London hospitals
}

\author{
A Thomson, $M$ Contreras, $S$ Knowles
}

\begin{abstract}
A retrospective audit of 200 transfusion episodes involving the use of platelets or fresh frozen plasma (FFP) was performed in five hospitals in London. It examined the currently used practices of transfusion and assessed the appropriateness of blood component treatment. It was necessary to search for an excess of case notes to provide a sufficient number of patients for review. In $61.5 \%$ of cases the reason for using the components was not stated. Inadequate documentation of the use of blood components occurred in $66 \%$ of cases. An accepted clinical indication for the use of components was evident in only $36 \%$ of the total; inappropriate use of FFP was particularly apparent.

It is concluded that many aspects of transfusion practice need to be improved.
\end{abstract}

The introduction of blood component treatment has had a major impact on the practice of transfusion, providing a larger number of therapeutic units from a single donation of blood. Specific replacement treatment for the patient's needs is provided and the safety and efficiency of blood transfusion is thereby enhanced. At the North London Blood Transfusion Centre (NLBTC), between 1979 and 1989, production of platelet concentrates increased from 42000 to 110000 units, and fresh frozen plasma for clinical use, from 7000 to 28000 units. But there is concern over this escalating demand for several reasons. Transfusion of blood components is not without risk, both from immunological adverse reactions and transfusion transmitted infection. Blood components, especially platelet concentrates, due to their short shelf-life, are frequently in limited supply and their appropriate use is required to ensure their availability for patients who really need them. Moreoever, during the past five years in the United Kingdom, despite efforts to recruit new donors, blood donation rates have remained static or, in some places, have even decreased. The collection and processing of blood components are also expensive, and cost containment in the health care system is necessary.

These concerns, coupled with previous reports of inappropriate use of blood components in other countries, ${ }^{1-4}$ prompted the decision to perform the present audit. The audit aimed to document some of the currently used practices of transfusion of blood components in hospitals supplied by the NLBTC. Results were then analysed to identify the areas which required change or intervention to encourage good transfusion practice.

\section{Methods}

Five of the major "user" hospitals of the NLBTC were chosen and, from each of these, 20 episodes of platelet transfusion and 20 of fresh frozen plasma (FFP) were reviewed-a total of 200 cases. All these transfusions occurred between October 1988 and November 1989. Initially, transfusion episodes were identified from the records of each hospital blood transfusion department, and the patients' notes were then sought from the medical records department. If the notes were not available no attempts were made to trace them to individual departments. Patients under the care of, or reviewed by, a haematologist were excluded as it was assumed that haematologists would use blood components according to recommended guidelines. Details of each hospital transfusion department's policy relating to the issue of platelets and FFP were recorded to determine what control, if any, was exercised over the use of blood components. A transfusion episode was defined as a 24 hour period during which blood and blood components were transfused.

Details recorded from the case notes included: (1) medical specialty; (2) diagnosis and surgical procedure; (3) reason for the transfusion; (4) adequacy of laboratory monitoring (platelet count or coagulation screen performed both before and after transfusion); (5) all units of blood and components transfused including recording of correct documentation-that is, an order for the blood or components signed by a medical officer, plus signatures of the persons administering the blood components at the time each unit was transfused; and (6) response to the transfusion.

When case notes were unavailable, although such patients were not included in the 200 cases in this study, details of the quantity of platelets or FFP used in the transfusion episodes and the medical specialty of each case were ascertained from the transfusion department records and from the hospital computer records. Using this information, reviewed cases were compared with unreviewed cases to determine whether they were representative of local practice.

Assessment of the appropriateness of the transfusion was made using the guidelines for
Transfusion Centre, Colindale Avenue, A Thomson M Contreras $S$ Knowles 
use of FFP and platelets from the National Institutes of Health Consensus Development Conferences (table 1). ${ }^{45}$ Cases were assigned into one of three categories: indicated, not indicated, or possibly indicated. The latter included those cases in which there was insufficient information to assess fully the episode, and also some situations in which the indication for the use of blood components is controversial.

\section{Results}

A total of 565 case notes had to be searched for

Table 1 NIH consensus conferences guidelines ${ }^{45}$

Summary of indications

Platelet transfusion treatment:

Thrombocytopenia or abnormal platelet function when bleeding or before invasive procedure

Prophylaxis when platelet count of $<20 \times 10^{9} / 1$

Massive transfusion

if thrombocytopenia documented and clinical bleeding evident

Not for prophylaxis during/after cardiac surgery

Fresh frozen plasma-indications:

Replacement of isolated factor deficiencies

when specific component therapy not available

Emergency reversal of warfarin effects

Massive transfusion

if documented hemostatic disorder and clinical bleeding evident

Antithrombin III deficiency ${ }^{\star}$

Thrombotic thrombocytopenic purpura

^Specific preparation now available

Table 2 Distribution of cases by specialty

\begin{tabular}{lcc}
\hline & $\begin{array}{l}\text { Percentage of } \\
\text { reviewed } \\
\text { cases }\end{array}$ & $\begin{array}{l}\text { Percentage of } \\
\text { total cases } \\
\text { initially sought }\end{array}$ \\
\hline Surgical: & & \\
Cardiothoracic & 43.0 & 23.7 \\
Vascular & 9.5 & 8.7 \\
General & 1.5 & 11.9 \\
Hepatobiliary & 5.5 & 5.7 \\
Urology/transplant & 2.5 & 4.4 \\
Neurosurgery & 3.0 & 2.1 \\
Orthopaedic & 2.0 & 2.1 \\
Obstetric & 1.0 & 1.4 \\
Medical: & 8.0 & 11.0 \\
General & 5.5 & 7.6 \\
Gastroenterology & 3.0 & 7.6 \\
Oncology & 4.0 & 2.7 \\
Intensive care & 1.5 & 3.4 \\
Renal & &
\end{tabular}

Table 3 Use of components-comparison of reviewed and unreviewed cases

\begin{tabular}{|c|c|c|c|c|}
\hline & \multicolumn{4}{|c|}{ Transfusion episodes } \\
\hline & \multicolumn{2}{|l|}{ Platelets } & \multicolumn{2}{|l|}{$F F P$} \\
\hline & Reviewed & Not reviewed & Reviewed & Not reviewed \\
\hline & \multicolumn{4}{|c|}{ Number of cases (number of units transfused) } \\
\hline $\begin{array}{l}\text { Surgical } \\
\text { Medical } \\
\text { Total } \\
\text { Average units/case }\end{array}$ & $\begin{array}{l}75(615) \\
25(175) \\
100(790) \\
7.9\end{array}$ & $\begin{array}{c}82(583) \\
78(562) \\
160(1145) \\
7 \cdot 1\end{array}$ & $\begin{array}{c}81(300) \\
19(87) \\
100(387) \\
3.9\end{array}$ & $\begin{array}{c}101(442) \\
61(208) \\
162(650) \\
4.0\end{array}$ \\
\hline
\end{tabular}

Table 4 Data obtained from 200 transfusion episodes audited

\begin{tabular}{|c|c|c|c|c|}
\hline & \multicolumn{4}{|c|}{ Transfusions } \\
\hline & Platelets & $F F P$ & Total & $(\%)$ \\
\hline $\begin{array}{l}1 \text { Reason for transfusion: } \\
\text { (a) documented } \\
\text { (b) deduced } \\
\text { (c) unknown } \\
2 \text { Correct documentation of all } \\
\text { blood and components } \\
3 \text { Adequate laboratory assessment: } \\
\text { (a) before transfusion } \\
\text { (b) after transfusion } \\
4 \text { Documentation of response to transfusion }\end{array}$ & $\begin{array}{r}47 \\
47 \\
6 \\
29 \\
\\
92 \\
94 \\
39\end{array}$ & $\begin{array}{r}30 \\
67 \\
3 \\
\\
37 \\
\\
81 \\
59 \\
31\end{array}$ & $\begin{array}{r}77 \\
114 \\
9 \\
66 \\
\\
173 \\
153 \\
70\end{array}$ & $\begin{array}{c}(38 \cdot 5) \\
(57) \\
(4 \cdot 5) \\
(33) \\
(86 \cdot 5) \\
(76 \cdot 5) \\
(35)\end{array}$ \\
\hline
\end{tabular}

to provide 200 cases for review. Three hundred and twenty two case notes were unavailable in the medical records departments, and of these, $98(17 \%$ of the total) had no tracer card to indicate their whereabouts. Forty three of the available cases were excluded as they were haematology cases.

Enforced selection bias was apparent in the reviewed cases, with underrepresentation in the number of cases from some medical specialties and an excess number of cardiothoracic cases (table 2). The reviewed cases were, however, representative quantitatively of local component use: the number of FFP per case averaged 3.9 in the reviewed group and 4.0 in the unreviewed group, and for the platelet transfusion episodes the figures were 7.9 units and $7 \cdot 1$ units, respectively (table 3 ).

Table 4 summarises some of the data collected. Many inadequacies were found. In $61.5 \%$ of cases the reason for the use of the components was not stated in the case notes, but from the available notes and laboratory request forms it could be deduced in $57 \%$ of the cases. The reasons for the use of the components are summarised in tables 5 and 6 .

Inadequate documentation of all blood and components transfused was found in $66 \%$ of patients.

Laboratory assessment before transfusion was either not performed, or the results not recorded in $13 \%$ of cases. Similarly, no posttransfusion assessment was found in $24 \%$. In $65 \%$ of cases there was no written record of the clinical response to the transfusion.

Assessment of the appropriateness of transfusion (table 7) showed disappointing results. Of the total, only $36 \%$ of cases were definitely indicated, with a further $24 \%$ possibly

Table 5. Reasons (stated or deduced) for use of platelets

\begin{tabular}{lc}
\hline Reason & $\begin{array}{l}\text { Number } \\
\text { of cases }\end{array}$ \\
\hline 1 Platelet count $<100 \times 10^{9} / 1:$ & \\
(a) intended surgery & 19 \\
(b) clinical bleeding & 18 \\
(c) massive transfusion & 6 \\
(d) neither (a), (b), (c) nor other complication & 9 \\
2 Platelet count normal or unknown: & 13 \\
(a) perioperative bleeding & 10 \\
(b) cardiopulmonary bypass $\dagger$ & 11 \\
(c) standard procedure/practice & 8 \\
(d) massive transfusion & 6 \\
(e) not stated/deduced & \\
\hline
\end{tabular}

* Seven of these patients were taking aspirin within the previous week.

tAll patients were taking aspirin within the previous week, but in only three was this given as the reason for the use of the platelets.

Table 6 Reasons (stated or deduced) for use of FFP

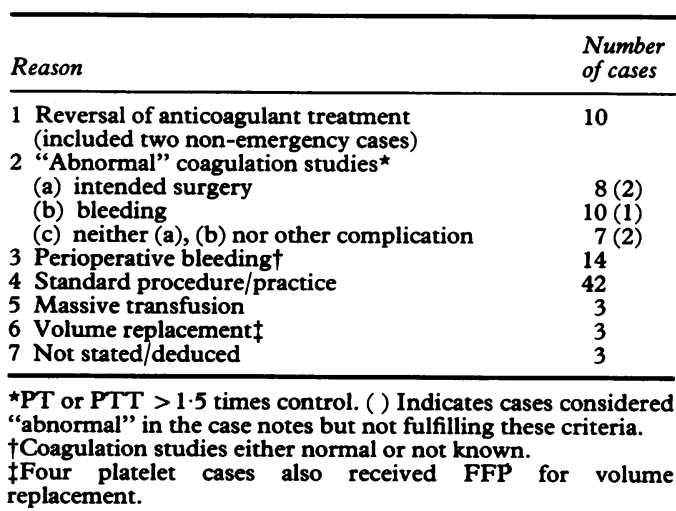


Table 7 Analysis of indication for transfusion of blood components in 200 cases

\begin{tabular}{llll}
\hline & \multicolumn{3}{l}{ Transfusion episodes } \\
\cline { 2 - 4 } Assessment & Platelets & FFP & Total \\
\hline Indicated & 52 & 21 & 73 \\
Not indicated & 19 & 60 & 79 \\
Possibly indicated & 29 & 19 & 48 \\
\hline
\end{tabular}

indicated. When the FFP and platelet cases were reviewed separately, FFP was used inappropriately in a much higher percentage of cases; $60 \%$ were not indicated. Some of the obvious situations in which FFP was misused included the following: use as part of a standard replacement regimen in a large number of patients, for example 1 unit of FFP for every 3-5 units of blood transfused; during or following cardiopulmonary bypass regardless of the haemostatic response; and as volume replacement instead of safer treatment with other colloid solutions. Platelet concentrates were also similarly inappropriately used in standard replacement regimens and routinely during cardiopulmonary bypass. Other unnecessary platelet use included transfusion to correct moderate thrombocytopenia in situations where there was no clinical bleeding or complication evident and no surgery or procedure planned. Overall, unnecessary use of platelets was, however, less evident: $19 \%$ of transfusions were not indicated.

As previously stated, a high proportion of the reviewed cases were cardiothoracic cases. In an attempt to account for the bias that these cases may have introduced, particularly into the assessment of the appropriateness of transfusion, the data were re-examined excluding all cardiothoracic cases. The resultant figures still confirmed that in $38 \%$ of cases FFP was inappropriately transfused and in $10 \%$ of cases platelets were misused.

None of the five hospitals had formal written guidelines governing the criteria for use of blood components. Orders of 2-4 units of FFP were issued without review in all hospitals, and in two hospitals the use of FFP according to standard replacement regimens-for example 1 unit of FFP for every 3-5 units of blood transfused-was permitted. Only where the use of FFP was considered by the staff in the transfusion laboratory to be excessive or unnecessary was referral to a haematologist requested. The use of platelets was under stricter control with, in all hospitals, requests usually needing the approval of the haematology registrar or senior registrar on call. Vetting of requests therefore depended on the experience and also availability of this person.

Performance of the individual hospitals was in most respects very similar. Differences did exist, however, in two particular areas. The first of these relates to the availability of case notes: two of the hospitals accounted for $92 \%$ of the case notes which had no tracer card to identify their position, and in both hospitals it was necessary to search for a larger number of case notes to obtain the required number of patients for review. The second aspect was that inappropriate use of both platelets and FFP was more apparent in one particular hospital

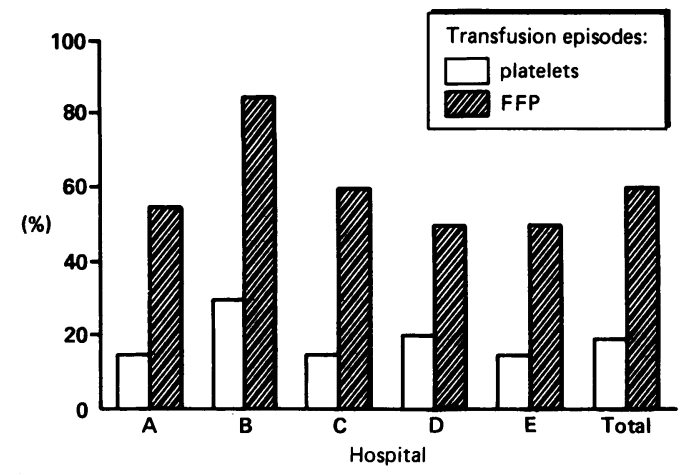

Inappropriate use of components: comparison of the five hospitals.

(figure). At this hospital the inappropriate use of FFP occurred in $85 \%$ of transfusion episodes as opposed to $60 \%$ overall in the five hospitals, and the equivalent figures for the inappropriate use of platelets were $30 \%$ and $19 \%$, respectively. These figures may be partly accounted for by the larger number of surgical specialty cases reviewed at this hospital.

\section{Discussion}

Several reasons justify the audit of transfusion practices. Blood component treatment has made available to clinicians a wide variety of choices and this has increased the potential for misuse because there is a variable level of knowledge of appropriate transfusion practices in the medical profession.

Our study agrees with studies performed in other countries, reporting that a significant proportion of the FFP and platelet concentrates which were transfused were given for inappropriate reasons. ${ }^{1-4}$

Overuse of blood components has been attributed to misconceptions of their value, lack of knowledge of the situations in which their use cannot be justified, and underestimation of the incidence and magnitude of possible complications. Educational programmes designed to address these misconceptions, combined with a concurrent audit of transfusion practices, have been shown to have a measurable effect on clinicians' attitudes and behaviour.$^{67}$ After such programmes a reduction in the number of blood components transfused, and also in the number of patients transfused for inappropriate reasons has been reported. ${ }^{6}$ No detrimental effects were noted.

This audit has highlighted that many areas of clinical practice need to be improved. In many cases the documentation of the transfusion episode, including the reason for the use of blood components, and the actual components transfused, was inadequate. A significant proportion of the patients' case notes were also unavailable. Both these factors cause concern. The ultimate fate of each unit of blood or blood component should be known and must be fully documented to comply with the Department of Health Circular on record keeping and stock control, ${ }^{8}$ and current regulations on product liability. ${ }^{9}$ If it became necessary to contact the recipient of a blood component from a donor possibly infectious for hepatitis B virus or HIV, it would be impossible to determine, with certainty, whether that unit was actually trans- 
fused to that specific recipient in two out of three of the cases reviewed.

If a blood transfusion recipient were to acquire HIV, then the consultant responsible for the recipient's care would, in many cases, have no written evidence to justify the use of the incriminated components. Litigation following on from transfusion-transmitted viral infection is unfortunately becoming common and the importance of adequate documentation is self evident.

Inappropriate use of the components, particularly FFP, was evident. The most important concern in this misuse is the exposure of patients to unnecessary risk. Another aspect, however, is the expenditure involved, which is important as cost containment in the health care system is necessary. For example, in the reviewed cases 194 units of FFP were inappropriately transfused. Each unit of FFP, using the Department of Health recommended charges for 1990 , costs $£ 14 \cdot 25$. The unnecessary expense in these 60 cases alone is evident and the potential wastage of resources which may be occurring is considerable.

It is likely that the abuse in the case of FFP is partly due to its easier availability. Little vetting on the use of FFP occurred in each of the hospitals; the use of platelets was under stricter control. Inadequate knowledge of the actual haemostatic value of blood components and the risks associated with their transfusion help to account for their unnecessary use. For example, many studies have been performed of the bleeding episodes which can be associated with cardiopulmonary bypass procedures, and in most instances bleeding has been related to platelet dysfunction rather than to a deficiency of plasma coagulation factors. ${ }^{1011}$ It is also now accepted that the routine use of FFP at or after cardiopulmonary bypass surgery exposes the patient to unnecessary risk and provides no known benefit. ${ }^{12}$ The use of FFP according to predetermined replacement regimens also cannot be justified, ${ }^{13}$ nor can its use as a volume replacement fluid, as there are other equally effective safer alternatives. Examples of such unnecessary use of FFP, however, in particular a high proportion of cases in which standard replacement regimens were followed, were common in this study.

Appropriate education is therefore of particular importance. The $B M J$ has recently published a much needed $A B C$ of Transfusion, ${ }^{14}$ and the NBTS has published a Handbook of Transfusion Medicine. ${ }^{15}$ National guidelines for the use of platelet concentrates and FFP are currently being formulated by the British Committee for Standards in Haematology and will be published later this year. These will provide an additional useful basis for education.

Educational programmes alone, however, have not been shown to produce a consistent change in clinical practice. ${ }^{16}$ It is also unlikely that isolated attempts, by staff of the hospital transfusion departments, to regulate the use of components will be wholly effective. To achieve significant improvement, involvement by representatives of all the specialties using blood transfusion is necessary. A hospital "transfusion committee" so formed can, through consensus, develop transfusion guidelines acceptable for local use, institute education programmes for all hospital blood users, and participate in ongoing medical audit to ensure the appropriate local use of blood and components. Such committees composed of surgeons, physicians, obstetricians, paediatricians, and haematologists are now mandatory in some countries. Establishment of transfusion committees initially in the five hospitals audited, and then in other hospitals in the North West Thames Region, is now underway. In future, assessment as to whether changes in practice have been effected by education and the work of the committees will also be valuable.

Although the transfusion practices reported in this audit will not be representative of other hospitals, they suggest that examination of local transfusion practice is necessary. The present audit will only be of value if its results provide the incentive for change and thereby promote future safe and effective practices of transfusion.

It has become increasingly difficult to collect enough blood from volunteer donors to meet the ever increasing demands for blood derivatives. In the United States of America a decline in collections of homologous blood has been offset by a decrease in transfusions of whole blood, red cells, and plasma. ${ }^{17}$ On the other hand, in England and Wales, demands for transfusion of blood derivatives are increasing, but this is not being matched by an increase in collections of homologous blood. Hopefully, education, audit, and the establishment of hospital transfusion committees will help us to curb misuse and realise that our current resources are sufficient to meet all justifiable demands.

1 Snyder AJ, Gottschall JL, Menitove JE. Why is fresh frozen plasma transfused? Transfusion 1986;26:107-12.

2 Shaikh BS, Wagar D, Lau PM, Campbell EW. Transfusion pattern of fresh frozen plasma in a medical school hospital. Vox Sang 1985;48:366-9.

3 Braunstein AH, Oberman HA. Transfusion of plasma components. Transfusion 1984;24:281-6.

4 Consensus Development Panel. National Institutes of Health: Fresh Frozen Plasma. Indications and risks. JAMA 1985;253:551-3.

5 Consensus Development Panel, National Institutes of Health: Platelet transfusion therapy. JAMA 1987; 257:1777-80.

6 Barnette RE, Fish DJ, Eisenstaedt RS. Modification of fresh frozen plasma transfusion practices through educational intervention. Transfusion 1990;30:253-7.

7 Simpson MB. Prospective-concurrent audits and medical consultation for platelet transfusions. Transfusion 1987; 27:192-5.

8 Department of Health. DHSS requirement for record keeping and stock control. HC(84)7. London: HMSO, 1984.

9 Consumer Protection Act 1987. Product Liability. Chapter 43, Part 1.

10 Harker LA, Malpass TW, Branson HE, Hessel EA II, Slichter SJ. Mechanism of abnormal bleeding in patients undergoing cardiopulmonary bypass: acquired transient undergoing cardiopulmonary bypass: acquired transient platelet dysfunction
$1980 ; 56: 824-34$.

11 Harker LA. Bleeding after cardiopulmonary bypass. $N \mathrm{Engl}$ $J$ Med 1986;314:1446-8.

12 Trimble AS, Osborn JJ, Kerthwood WJ, Gerbode F. The prophylactic use of fresh frozen plasma after extracorporeal circulation. $J$ Thorac Cardiovasc Surg 1964;48: 314-6.

13 Mannucci PM, Federici AB, Sirchia G. Haemostasis testing during massive blood replacement. Vox Sang 1982; 42:113-23.

14 Marcela Contreras, ed. $A B C$ of transfusion. London: BMJ, 1990.

15 McClelland DBL, ed. Handbook of transfusion medicine. United Kingdom Blood Transfusion Service. London: United Kingdom Blood Transfusion

16 Brien WF, Butler RJ, Inwood MJ. An audit of blood component therapy in a Canadian general teaching hoscomponent therapy in a Canadian general
pital. Can Med Assoc $J 1989 ; 140: 812-5$.

17 Surgenor DM, Wallace EL, Steven HS, Hao MBA, Chapman BA. Collection and transfusion of blood in the United States, 1982-1988. N Engl J Med 1990;322: 1646-51. 